\title{
TIME-FREQUENCY PARTITIONS FOR THE GELFAND TRIPLE $\left(S_{0}, L^{2}, S_{0}^{\prime}\right)$
}

\author{
MONIKA DÖRFLER, HANS G. FEICHTINGER and KARLHEINZ GRÖCHENIG*
}

\begin{abstract}
We give a new characterization of the Gelfand triple of function spaces in $\left(S_{0}, L^{2}, S_{0}^{\prime}\right)$ by means of a family of time-frequency localization operators. The localization operators are defined by the short-time Fourier transform and determine the local time-frequency behavior, whereas the global time-frequency distribution is characterized by a sequence space norm. We also show that the alternative time-frequency localization method with the Weyl transform fails to yield a similar characterization of time-frequency distribution.
\end{abstract}

\section{Introduction}

In the perception of music, speech, and any other audio signals, the separation of signal components in the time-frequency domain is a natural ability that is performed unconsciously by the human ear. For instance, a melody played by a flute and its accompaniment by a piano can be discriminated easily by most listeners. In an engineering context, this process can be described as the separation of signal components in the time-frequency domain by means of a time-variant filtering procedure. An analogous problem occurs in the quantum mechanics of many-particle systems. Given a suitable phase-space representation of a quantum mechanical state, one would like to assign a certain region in phase-space to each particle.

The mathematical task of extraction and localization of signal components (or particle separation in phase-space) is highly non-trivial. This is a consequence of the uncertainty principle, which implies that no signal can have compact support in the time-frequency domain. Thus, in a mathematical sense, two signals can never be clearly discerned and perfectly separated by their time-frequency characteristics.

In this paper we investigate a simple model for signal/particle separation that is based on time-frequency localization operators and time-frequency analysis. In this model we will characterize the global time-frequency distribution

\footnotetext{
* The first author has been supported by the Austrian science fund FWF, Projects P14485 and P15605.

Received July 28, 2004.
} 
by means of the local components. Although this model requires further refinements, it already leads to interesting mathematical problems, and these will be our subject here.

As our main tool for time-frequency analysis we use the short-time Fourier transform (STFT, with slightly different normalizations also called coherent state transform, ambiguity function, or Wigner distribution), and the associated time-frequency localization operators. The short-time Fourier transform of a function $f \in L^{2}\left(\mathrm{R}^{d}\right)$ with respect to a window function $\varphi \in L^{2}\left(\mathrm{R}^{d}\right)$ is defined as

$$
\mathscr{V}_{\varphi} f(x, \omega)=\int_{\mathrm{R}^{d}} f(t) \bar{\varphi}(t-x) e^{-2 \pi i \omega \cdot t} d t=\left\langle f, M_{\omega} T_{x} \varphi\right\rangle,
$$

where $T_{x} f(t)=f(t-x)$ and $M_{\omega} f(t)=e^{2 \pi i \omega \cdot t} f(t)$ are translation and modulation operators, respectively. We will denote a time-frequency shift by $\pi(z)=M_{z_{2}} T_{z_{1}}$ for $z=\left(z_{1}, z_{2}\right) \in \mathrm{R}^{2 d}$.

Since $\mathscr{V}_{\varphi} f(z)$ measures the time-frequency content near the point $z$ in the time-frequency plane $\mathrm{R}^{2 d}$, we would like to construct a projection operator whose outcome has exactly the STFT $\mathscr{V}_{\varphi}\left(P_{\Omega} f\right)=\chi_{\Omega} \cdot \mathscr{V}_{\varphi} f$. However, a STFT can never have support of finite measure [19]. In the literature one therefore resorts to the following definition of localization operators which are defined in terms of the STFT.

DEFINITION 1.1 (Time-frequency localization operator). Let $\varphi$ be a given window-function and $\sigma$ a bounded non-negative function on $\mathrm{R}^{2 d}$. Then the time-frequency localization operator $H_{\sigma}$ corresponding to the window $\varphi$ and the symbol $\sigma$ is formally defined as

$$
H_{\sigma} f=\int_{\mathrm{R}^{2 d}} \sigma(z) \mathscr{V}_{\varphi} f(z) \pi(z) \varphi d z
$$

If $\sigma \equiv 1$ and $\|\varphi\|_{2}=1$, then $\int_{\mathrm{R}^{2 d}} \mathscr{V}_{\varphi} f(z) \pi(z) \varphi d z=f$ (inversion formula for the STFT), and so $H_{\sigma}$ coincides with the identity. If $\sigma$ has compact support in $\Omega \subseteq \mathrm{R}^{d}$, then $H_{\sigma} f$ can be interpreted as the part of $f$ that essentially lives on $\Omega$ in the time-frequency plane, hence the term time-frequency localization operator. Thus the function $H_{\sigma} f$ comes as close to being the projection of $f$ onto the region $\Omega$ in the time-frequency plane as is compatible with the uncertainty principle. See [8] for a precise statement.

Operators of this kind have been introduced and studied by Daubechies [6], Ramanathan and Topiwala [21], and Wong [22]. They occur in signal analysis, in quantization procedures in physics [2], or in the approximation of pseudodifferential operators [5]. 
Next we may consider an entire collection of localization operators $\left\{H_{T_{k} \sigma}\right.$ : $\left.k \in \mathbf{Z}^{2 d}\right\}$. Then the function-valued mapping $f \rightarrow\left\{H_{T_{k} \sigma} f\right\}$ can be interpreted as a decomposition of $f$ into its signal components $H_{T_{k} \sigma} f$ living essentially on $\operatorname{supp} T_{k} \sigma=k+\operatorname{supp} \sigma$ in the time-frequency plane, and each norm $\left\|H_{T_{k} \sigma} f\right\|_{2}^{2}$ is the energy of this signal component. Conversely, the global time-frequency distribution of $f$ should be completely encoded in the sequence $\left\{\left\|H_{T_{k} \sigma}\right\|_{2}\right.$ : $k \in \mathrm{Z}^{2 d}$.

Our main theorem demonstrates that this intuitive picture of localization operators and signal separation is by-and-large correct. For the exact formulation we need the norm $\|f\|_{S_{0}}=\int_{\mathrm{R}^{2 d}}\left|\mathscr{V}_{\varphi} f(z)\right| d z$, which measures "good" time-frequency concentration. The dense embeddings $S_{0}\left(\mathrm{R}^{d}\right) \hookrightarrow L^{2}\left(\mathrm{R}^{d}\right) \hookrightarrow$ $S_{0}^{\prime}\left(\mathrm{R}^{d}\right)$ show that the triple of spaces $\left(S_{0}\left(\mathrm{R}^{d}\right), L^{2}\left(\mathrm{R}^{d}\right), S_{0}^{\prime}\left(\mathrm{R}^{d}\right)\right)$ is an example of a so-called Gelfand triple, see [16].

The main statement can now be formulated as follows:

THEOREM 1.2. Assume that the symbol $\sigma$ is a non-negative bounded function with compact support on $\mathrm{R}^{2 d}$ such that

$$
0<A \leq \sum_{k \in Z^{2 d}} T_{k} \sigma(z) \leq B \quad \forall z \in \mathrm{R}^{2 d} .
$$

Then the mapping $f \mapsto\left(\left\|H_{T_{k} \sigma} f\right\|_{2}\right)_{k \in Z^{2 d}}$ is continuous from $\left(S_{0}, L^{2}, S_{0}^{\prime}\right)$ to $\left(\ell^{1}, \ell^{2}, \ell^{\infty}\right)$. Conversely, there exists a constant $C>0$ such that

$$
\|f\|_{S_{0}} \leq C \sum_{k \in \mathbf{Z}^{2 d}}\left\|H_{T_{k} \sigma} f\right\|_{2}, \quad\|f\|_{2}^{2} \leq C^{2} \sum_{k \in Z^{2 d}}\left\|H_{T_{k} \sigma} f\right\|_{2}^{2},
$$

and $\|f\|_{S_{0}^{\prime}} \leq C \sup _{k \in \mathrm{Z}^{2 d}}\left\|H_{T_{k} \sigma} f\right\|_{2}$.

This paper is organized as follows. The Gelfand triple will be introduced in Section 2. We recall some important results about Gabor frames and the Zak transform, as these will be the main tools in the proof of Theorem 1.2. Several preliminary results on the localization operators will be given. In Section 3 we prove the main statement and formulate some of its consequences. In the last section we consider an alternative model of time-frequency localization, namely the Weyl transform and show that a corresponding version of Theorem 1.2 fails. We finally remark on generalizations and future work.

\section{Preliminaries}

We collect a few concepts and facts about Gabor frames and the Zak transform. The statements are well-known and already treated in the textbooks [7], [17] or in various chapters of [13]. 


\subsection{The Gelfand triple $\left(S_{0}, L^{2}, S_{0}^{\prime}\right)$}

Definition 2.1. Fix a non-zero "window" function $\varphi \in \mathscr{S}\left(\mathrm{R}^{d}\right)$. The space $S_{0}\left(\mathrm{R}^{d}\right)$ is given by

$$
S_{0}\left(\mathrm{R}^{d}\right)=\left\{f \in L^{2}\left(\mathrm{R}^{d}\right):\|f\|_{S_{0}}:=\left\|\mathscr{V}_{\varphi} f\right\|_{L^{1}\left(\mathrm{R}^{2 d}\right)}<\infty\right\} .
$$

The following Proposition summarizes some properties of $S_{0}$ and its dual, the distribution space $S_{0}^{\prime}$.

Proposition 2.2. $S_{0}\left(\mathbf{R}^{d}\right)$ is a Banach space and densely embedded in $L^{2}\left(\mathrm{R}^{d}\right)$. The definition of $S_{0}$ is independent of the window $\varphi \in \mathscr{S}$, and different choices of $\varphi \in \mathscr{S}$ yield equivalent norms on $S_{0}$.

By duality, $L^{2}\left(\mathrm{R}^{d}\right)$ is densely and weak ${ }^{*}$-continuously embedded in $S_{0}^{\prime}\left(\mathrm{R}^{d}\right)$ and can also be characterized by the norm $\|f\|_{S_{0}^{\prime}}=\left\|\mathscr{V}_{\varphi} f\right\|_{L^{\infty}}$.

In general, a triple of spaces $\left(\mathscr{B}, \mathscr{H}, \mathscr{B}^{\prime}\right)$ is called a Gelfand triple [16], if $\mathscr{H}$ is a Hilbert space and $\mathscr{B}$ a Banach space with dual space $\mathscr{B}^{\prime}$, such that $\mathscr{B}$ is densely embedded into $\mathscr{H}$ and $\mathscr{H}$ is weak*-dense in $\mathscr{B}^{\prime}$. Proposition 2.2 thus states that the three spaces $\left(S_{0}, L^{2}, S_{0}^{\prime}\right)$ form a Gelfand triple.

For a proof, equivalent characterizations, and more results on $S_{0}$ we refer to [10], [15],[12].

\subsection{Gabor frames}

Let $\mathscr{G}(\varphi, a, b)$ denote a set of functions $\left\{M_{m b} T_{n a} \varphi: m, n \in Z^{d}\right\}$ in $L^{2}\left(\mathrm{R}^{d}\right)$, where $a, b>0$ are the time- and frequency shift parameters. The operator

$$
S_{\varphi} f=\sum_{m, n \in Z^{d}}\left\langle f, M_{m b} T_{n a} \varphi\right\rangle M_{m b} T_{n a} \varphi
$$

is the frame operator corresponding to $\mathscr{G}(\varphi, a, b)$. If $S_{\varphi}$ is invertible, $\mathscr{G}(\varphi, a, b)$ is a Gabor frame for $L^{2}(\mathrm{R})$. In this case, $\left\|S_{\varphi}\right\|_{o p}$ and $\left\|S_{\varphi}^{-1}\right\|_{o p}^{-1}$ are the upper and lower frame bounds. Note that the frame coefficients of $\left[\left\langle f, M_{b n} T_{a m} \varphi\right\rangle\right]_{m, n}$ correspond to a sampling of the STFT on the lattice $\Lambda=a \mathbf{Z}^{d} \times b \mathbf{Z}^{d}$.

If we use several windows $\varphi_{j}, j=1, \ldots, r$, and consider the union $\bigcup_{j=1}^{r} \mathscr{G}\left(\varphi_{j}, a, b\right)$, we speak of a multi-window Gabor frame, whenever its frame operator given by

$$
S f=\sum_{j=1}^{r} \sum_{m, n \in \mathbb{Z}^{d}}\left\langle f, M_{m b} T_{n a} \varphi_{j}\right\rangle M_{m b} T_{n a} \varphi_{j}=\sum_{j=1}^{r} S_{\varphi_{j}} f,
$$

is invertible. 
The reconstruction from the frame coefficients $\left[\left\langle f, M_{b m} T_{a n} \varphi_{j}\right\rangle\right]_{j, m, n}$ is possible by means of a dual frame. The canonical dual frame is given by $S^{-1} M_{b m} T_{a n} \varphi_{j}=M_{b m} T_{a n} S^{-1} \varphi_{j}:=M_{b m} T_{a n} \gamma_{j}$ (since the frame operator $S$ commutes with time-frequency shifts on the lattice $a Z^{d} \times b Z^{d}$ ). Then we have

$$
\begin{aligned}
f & =S^{-1} S f=\sum_{j, m, n}\left\langle f, M_{b m} T_{a n} \varphi_{j}\right\rangle M_{b m} T_{a n} \gamma_{j} \\
& =S S^{-1} f=\sum_{j, m, n}\left\langle f, M_{b m} T_{a n} \gamma_{j}\right\rangle M_{b m} T_{a n} \varphi_{j} .
\end{aligned}
$$

Thus the knowledge of the (canonical) dual windows $\gamma_{j}=S^{-1} \varphi_{j}$ and their properties is crucial for the reconstruction.

\subsection{The Zak Transform}

In this paper we consider only the case of critical sampling $a b=1$. By applying a dilation, we may restrict without loss of generality to $a=b=1$. From now on, we will assume that the lattice is $Z^{d} \times Z^{d}$.

In this case we can apply Zak transform methods to the analysis of Gabor systems, because the Zak transform diagonalizes the frame operator in the case of critical sampling.

The Zak transform $Z f$ of a function $f$ on $\mathrm{R}^{d}$ is defined by

$$
Z f(x, \omega)=\sum_{k \in Z^{d}} f(x-k) e^{2 \pi i k \cdot \omega},
$$

whenever the sum makes sense.

Proposition 2.3. (i) The Zak transform maps the time-frequency shift operators $T_{k}$ and $M_{l}$ to multiplication operators:

$$
Z\left(M_{l} T_{k} f\right)(x, \omega)=e^{2 \pi i(l \cdot x-k \cdot \omega)} Z f(x, \omega)
$$

for $k, l \in \mathbf{Z}^{d}$.

(ii) The Zak transform can be extended to a unitary operator from $\mathbf{L}^{2}\left(\mathbf{R}^{d}\right)$ onto $\mathbf{L}^{2}\left([0,1]^{d} \times[0,1]^{d}\right)$.

(iii) If $f \in S_{0}$, then $Z f$ is uniformly continuous on $\mathrm{R}^{2 d}$ and $|Z f|^{2}$ is a periodic function (with period $\mathrm{Z}^{2 d}$ ) with an absolutetly convergent Fourier series.

(iv) If $f \in S_{0}\left(\mathrm{R}^{d}\right)$, then $Z f$ must have a zero on $[0,1]^{2 d}$.

For a proof of Proposition 2.3 see [17, Section 8.2] and [19]. As a consequence of (2), the Zak transform diagonalizes the frame operator on $Z^{2 d}$ and thus leads to a useful characterization of Gabor frames. 
Proposition 2.4. Let $\varphi_{j} \in \mathbf{L}^{2}\left(\mathbf{R}^{d}\right)$ for $j=1, \ldots, r$. Then $\bigcup_{j=1}^{r} \mathscr{G}\left(\varphi_{j}, \mathrm{Z}^{2 d}\right)$ is a multi-window Gabor frame if and only if

$$
0<A \leq \sum_{j=1}^{r}\left|Z \varphi_{j}(x, \omega)\right|^{2} \leq B<\infty \quad \text { a.a. } \quad x, \omega .
$$

Proof. The Zak transform diagonalizes the frame operator $S$ in the sense that

$$
Z\left(S_{\varphi} f\right)=|Z \varphi|^{2} Z f
$$

see, for example, [1] or [17, Theorem 8.3.1]. Since the frame operator of a multi-window Gabor frame can be written as $S=\sum_{j} S_{\varphi_{j}}$, we have $Z(S f)=$ $\left(\sum_{j=1}^{r}\left|Z \varphi_{j}\right|^{2}\right) Z f$. Consequently, the operator inequality $A I \leq S \leq B I$ is equivalent to (3), and thus $S$ is bounded and invertible if and only if (3) holds.

We now state a characterization of $S_{0}$ and $S_{0}^{\prime}$ by means of multi-window Gabor frames.

LeMmA 2.5. Let $\left\{\pi(k) \varphi_{j}, k \in \mathrm{Z}^{d} \times \mathrm{Z}^{d}, j=1, \ldots, r\right\}$ be a multi-window Gabor frame and assume that $\varphi_{j} \in S_{0}\left(\mathrm{R}^{d}\right)$ for all $j$. Then the dual windows $\gamma_{j}$ are also in $S_{0}\left(\mathbf{R}^{d}\right)$. As a consequence, $f$ belongs to $S_{0}\left(\mathbf{R}^{d}\right)$ if and only if each coefficient sequence

$$
\left(\left\langle f, \pi(k) \varphi_{j}\right\rangle\right)_{k \in \mathrm{Z}^{2 d}}
$$

for $j=1, \ldots, r$, belongs to $\ell^{1}\left(\mathrm{Z}^{2 d}\right)$. Furthermore, the norm-equivalence

$$
C_{1}\|f\|_{S_{0}} \leq \sum_{j=1}^{r} \sum_{k \in Z^{2 d}}\left|\left\langle f, \pi(k) \varphi_{j}\right\rangle\right| \leq C_{2}\|f\|_{S_{0}}
$$

holds on $S_{0}\left(\mathrm{R}^{d}\right)$.

Analogously, $f \in S_{0}^{\prime}\left(\mathbf{R}^{d}\right)$ if and only if each coefficient sequence (5) is in $\ell^{\infty}\left(\mathrm{Z}^{2 d}\right)$ for $j=1, \ldots, r$, with the corresponding norm-equivalence.

Proof. It suffices to show that the dual windows $\gamma_{j}$ are in $S_{0}$. The characterization of $S_{0}$ and $S_{0}^{\prime}$ and the norm-equivalences then follow from Theorem 3.3.1 and Corollary 3.3.2 in [15] or [11].

Since the dual windows $\gamma_{j}$ are given by $\gamma_{j}=S^{-1} \varphi_{j}$, Proposition 2.4 implies that the Zak transform of $\gamma_{j}$ is

$$
Z \gamma_{j}=Z\left(S^{-1} \varphi_{j}\right)=\left(\sum_{j=1}^{r}\left|Z \varphi_{j}\right|^{2}\right)^{-1} Z \varphi_{j}
$$


Since by Proposition 2.3 (iii) each $\left|Z \varphi_{j}\right|^{2}$ has an absolutely convergent Fourier series, we conclude from Wiener's Lemma that $\left(\sum_{j=1}^{r}\left|Z \varphi_{j}\right|^{2}\right)^{-1}$ has also an absolutely convergent Fourier series, which we may write as

$$
\left(\sum_{j=1}^{r}\left|Z \varphi_{j}\right|^{2}\right)^{-1}(x, \omega)=\sum_{k, l \in Z^{d}} a_{k l} e^{2 \pi i(k \cdot x+l \cdot \omega)}
$$

for some coefficient sequence $\left(a_{k l}\right) \in \ell^{1}$ (depending, of course, on $j$ ). Hence, by (2), we have

$$
Z \gamma_{j}=\sum_{k, l \in Z^{d}} a_{k l} e^{2 \pi i(k \cdot x+l \cdot \omega)} Z \varphi_{j}=\sum_{k, l \in Z^{d}} a_{k l} Z\left(M_{k} T_{-l} \varphi_{j}\right)
$$

which yields $\gamma_{j}=\sum_{k, l \in Z^{d}} a_{k l} M_{k} T_{-l} \varphi_{j}$. Consequently,

$$
\left\|\gamma_{j}\right\|_{S_{0}} \leq \sum_{k, l \in Z^{d}}\left|a_{k l}\right|\left\|M_{k} T_{-l} \varphi_{j}\right\|_{S_{0}}=\left\|\varphi_{j}\right\|_{S_{0}}\|a\|_{1}
$$

and $\gamma_{j} \in S_{0}$ for all $j=1, \ldots, r$.

\subsection{Properties of the Localization Operators}

Next we discuss some elementary properties of the localization operators $H_{\sigma}$. For an introduction to the general theory we refer to [22], for a detailed study of boundedness and Schatten class properties to [4].

LEMma 2.6 (Intertwining property). If $\sigma \in L^{\infty}, \varphi \in L^{2}$, and $k \in \mathrm{R}^{2 d}$, then

$$
\pi(k) H_{\sigma} \pi(k)^{*}=H_{T_{k} \sigma} .
$$

Proof. Write $z=\left(z_{1}, z_{2}\right), k=\left(k_{1}, k_{2}\right)$, then $\pi(k) \pi(z)=e^{-2 \pi i k_{1} z_{2}} \pi(z+$ $k)$ and $\mathscr{V}_{\varphi}\left(\pi^{*}(k) f\right)=\left(\mathscr{V}_{\varphi} f\right)(z+k) e^{2 \pi i k_{1} z_{2}}$. Therefore, as a vector-valued integral,

$$
\begin{aligned}
\pi(k) H_{\sigma} \pi^{*}(k) f & =\int_{\mathrm{R}^{2 d}} \sigma(z) \mathscr{V}_{\varphi}\left(\pi^{*}(k) f\right)(z) \pi(k) \pi(z) \varphi d z \\
& =\int_{\mathrm{R}^{2 d}} e^{2 \pi i k_{1} z_{2}} \sigma(z) \mathscr{V}_{\varphi} f(z+k) e^{-2 \pi i k_{1} z_{2}} \pi(z+k) \varphi d z \\
& =\int_{\mathrm{R}^{2 d}} \sigma(z-k) \mathscr{V}_{\varphi} f(z) \pi(z) \varphi d z=H_{T_{k} \sigma} f .
\end{aligned}
$$


For estimates of the STFT of $H_{\sigma} f$ we introduce the formal adjoint of $\mathscr{V}_{\varphi}$, namely

$$
\mathscr{V}_{\varphi}^{*} F=\int_{\mathrm{R}^{2 d}} F(z) \pi(z) \varphi d z
$$

which maps functions on $\mathrm{R}^{2 d}$ to functions or distributions on $\mathrm{R}^{d}$. With the adjoint mapping we can write the localization operators as

$$
H_{\sigma} f=\mathscr{V}_{\varphi}^{*}\left(\sigma \mathscr{V}_{\varphi} f\right)
$$

The STFT of $\mathscr{V}_{\varphi}^{*} F$ satisfies a fundamental pointwise estimate [17, Proposition 11.3.2.]:

$$
\left|\mathscr{V}_{\varphi}\left(\mathscr{V}_{\varphi}^{*} F\right)(z)\right| \leq\left(\left|\mathscr{V}_{\varphi} \varphi\right| *|F|\right)(z) \quad \forall z \in \mathrm{R}^{2 d}
$$

This estimate is extremely useful for the derivation of norm estimates.

In the following we fix a non-negative symbol $\sigma$ and investigate the set of operators $\left\{H_{T_{k} \sigma}: k \in \mathrm{Z}^{2 d}\right\}$. To simplify notation we will therefore write $H_{k}$ instead of $H_{T_{k} \sigma}$, in particular, $H_{0}=H_{\sigma}$ by some abuse of notation.

Lemma 2.7. (i) Assume that $\sigma \in L^{1}\left(\mathrm{R}^{2 d}\right), \sigma \geq 0$ and that $\varphi \in L^{2}\left(\mathrm{R}^{d}\right)$. Then each $H_{k}, k \in \mathrm{Z}^{2 d}$, is a positive trace-class operator.

(ii) If, in addition, $\varphi \in S_{0}\left(\mathrm{R}^{d}\right)$, then each $H_{k}$ is bounded from $S_{0}^{\prime}$ into $S_{0}$ (such operators are called regularizing). In particular, all eigenfunctions $\varphi_{j}$ of $H_{0}$ belong to $S_{0}$.

Proof. Statement (i) is well-known and has been proved many times, e.g., in [3], [14], [22].

(ii) To show the regularizing property, we first use (6)

$$
\left|\mathscr{V}_{\varphi} \mathscr{V}_{\varphi}^{*}\left(\sigma \mathscr{V}_{\varphi} f\right)\right| \leq\left|\mathscr{V}_{\varphi} \varphi\right| *\left|\sigma \mathscr{V}_{\varphi} f\right|
$$

Hence, for $f \in S_{0}^{\prime}$ we obtain

$$
\begin{aligned}
\left\|H_{\sigma} f\right\|_{S_{0}} & =\left\|\mathscr{V}_{\varphi} \mathscr{V}_{\varphi}^{*}\left(\sigma \mathscr{V}_{\varphi} f\right)\right\|_{1} \\
& \leq\left\|\left|\mathscr{V}_{\varphi} \varphi\right| *\left|\sigma \mathscr{V}_{\varphi} f\right|\right\|_{1} \\
& \leq\left\|\mathscr{V}_{\varphi} \varphi\right\|_{1}\left\|\sigma \mathscr{V}_{\varphi} f\right\|_{1}
\end{aligned}
$$

Since $\varphi \in S_{0}$ if and only if $\mathscr{V}_{\varphi} \varphi \in L^{1}\left(\mathrm{R}^{2 d}\right)$ by [17, Prop. 12.1.2], we find that

$$
\left\|H_{\sigma} f\right\|_{S_{0}} \leq C\|\sigma\|_{1}\left\|\mathscr{V}_{\varphi} f\right\|_{\infty}=C\|\sigma\|_{1}\|f\|_{S_{0}^{\prime}}
$$

and thus $H_{k}$ is bounded from $S_{0}^{\prime}$ to $S_{0}$. 
Applying the spectral theorem for compact selfadjoint operators, we obtain the following spectral representation of $H_{k}=H_{T_{k} \sigma}$.

COROLlaRY 2.8. There exists a positive sequence of eigenvalues $\mathbf{c}=\left(c_{j}\right) \in$ $\ell^{1}$ and an orthonormal system of eigenfunctions $\varphi_{j} \in S_{0}$, such that

$$
H_{0} f=\sum_{j=1}^{\infty} c_{j}\left\langle f, \varphi_{j}\right\rangle \varphi_{j}
$$

It follows that

$$
H_{k} f=H_{T_{k} \sigma} f=\pi(k) H_{\sigma} \pi(k)^{*} f=\sum_{j=1}^{\infty} c_{j}\left\langle f, \pi(k) \varphi_{j}\right\rangle \pi(k) \varphi_{j},
$$

where $\left\{\pi(k) \varphi_{j}: j \in \mathbf{N}\right\}$ is an orthonormal system of eigenfunctions of $H_{k}$.

\section{The Main Statement}

The following result gives a precise meaning to the intuition that $H_{k} f$ is the component of $f$ that "lives" near $k$ in the time-frequency plane.

THeOREM 3.1. Let $\sigma \in L^{1}\left(\mathbf{R}^{2 d}\right)$ be a non-negative symbol satisfying the condition

$$
A \leq \sum_{k \in Z^{2 d}} T_{k} \sigma \leq B, \quad \text { a.e. }
$$

for two constants $A, B>0$ and assume that $\varphi \in S_{0}\left(\mathrm{R}^{d}\right)$.

Then $f \in S_{0}$ if and only if

$$
\sum_{k \in Z^{2 d}}\left\|H_{k} f\right\|_{2}<\infty
$$

and (12) defines an equivalent norm on $S_{0}\left(\mathrm{R}^{d}\right)$. Similarly, we obtain the normequivalences

$$
\|f\|_{S_{0}^{\prime}} \asymp \sup _{k \in Z^{2 d}}\left\|H_{k} f\right\|_{2}
$$

and

$$
\|f\|_{2}^{2} \asymp \sum_{k \in Z^{2 d}}\left\|H_{k} f\right\|_{2}^{2}
$$

to characterize $S_{0}^{\prime}$ and $L^{2}$. 
The statements of Theorem 3.1 can be recast by using the notion of Gelfand triples.

CoROllary 3.2. The map $f \mapsto\left(\left\|H_{k} f\right\|_{2}\right)_{k \in Z^{2 d}}$ is an isomorphism between the Gelfand triples $\left(S_{0}, L^{2}, S_{0}^{\prime}\right)$ onto a closed subspace of $\left(\ell^{1}, \ell^{2}, \ell^{\infty}\right)$.

By interpolation we obtain a characterization of the modulation spaces $M^{p}\left(\mathrm{R}^{d}\right)$. These are the Banach spaces defined by the norm $\|f\|_{M^{p}}=\left\|\mathscr{V}_{\varphi} f\right\|_{p}$, and can also be characterized as interpolation spaces between $S_{0}=M^{1}$ and $S_{0}^{\prime}=M^{\infty}$ as $\left[S_{0}, S_{0}^{\prime}\right]_{\theta}=M^{p}$.

COROLlaRY 3.3. Under the assumptions of Theorem 3.1, there exist constants $C_{1}>0$ and $C_{2}>0$, such that for all $f \in M^{p}\left(\mathrm{R}^{d}\right)$ :

$$
\begin{aligned}
C_{1}\left(\sum_{k \in \mathrm{Z}^{2 d}}\left\|H_{k} f\right\|_{2}^{p}\right)^{\frac{1}{p}} \leq\left(\int_{\mathrm{R}^{2 d}}\left|\mathscr{V}_{\varphi} f(z)\right|^{p} d z\right)^{\frac{1}{p}} & \\
& =\|f\|_{M^{p}} \leq C_{2}\left(\sum_{k \in \mathrm{Z}^{2 d}}\left\|H_{k} f\right\|_{2}^{p}\right)^{\frac{1}{p}} .
\end{aligned}
$$

The following auxiliary result will be needed in the proof of Theorem 3.1.

LEMma 3.4. Let $\left\{\varphi_{j}: j \in \mathbf{N}\right\}$ be the orthonormal system of eigenfunctions of $H_{0}$. Then there exists a finite subset $\left\{\varphi_{j}: j=1, \ldots r\right\}$ of eigenfunctions such that the family $\left\{\pi(k) \varphi_{j}: j=1, \ldots, r, k \in \mathrm{Z}^{2 d}\right\}$ is a multi-window Gabor frame for $L^{2}\left(\mathrm{R}^{d}\right)$.

Proof. By Proposition 2.4, the set $\left\{\pi(k) \varphi_{j}: k \in \mathrm{Z}^{2 d}, j=1, \ldots, r\right\}$ is a multi-window Gabor frame if and only if the expression $\sum_{j=1}^{r}\left|Z \varphi_{j}(x, \omega)\right|^{2}$ is bounded above and below.

The upper bound $\sum_{j=1}^{r}\left|Z \varphi_{j}\right|^{2} \leq B$ is clear, since for $\varphi_{j} \in S_{0}$ all Zak transforms $Z \varphi_{j}$ are continuous on $[0,1]^{2 d}$ by Proposition 2.3 (iii). The continuity of $Z \varphi_{j}$ further implies that a lower bound $\sum_{j=1}^{r}\left|Z \varphi_{j}\right|^{2} \geq A>0$ holds if and only if the $Z \varphi_{j}$ 's do not have a common zero.

Let $\mathscr{Z}_{j}=\left\{\xi \in[0,1]^{2 d}: Z \varphi_{j}(\xi)=0\right\}$ be the set of zeros of $Z \varphi_{j}$. We first show by contradiction that $\bigcap_{j=1}^{\infty} \mathscr{Z}_{j}=\emptyset$. Assume that there exists a $\xi=\left(\xi_{1}, \xi_{2}\right) \in[0,1]^{2 d}$ such that $Z \varphi_{j}(\xi)=0$ for all $j \in \mathrm{N}$. Then we may define a non-zero functional $f$ by

$$
\langle f, \varphi\rangle=Z \varphi(\xi) \quad \forall \varphi \in S_{0} .
$$

Since by a lemma in [10] or [17, Prop. 12.1.4b]

$$
|Z \varphi(\xi)| \leq \sum_{k \in Z^{d}} \sup _{x \in[0,1]^{d}}|\varphi(x-k)| \leq C\|\varphi\|_{S_{0}},
$$


the functional $f$ is in $S_{0}^{\prime}\left(\mathbf{R}^{d}\right)$. Using Proposition 2.3(i), we obtain

$$
\left\langle f, \pi\left(k_{1}, k_{2}\right) \varphi_{j}\right\rangle=Z\left(\pi\left(k_{1}, k_{2}\right) \varphi_{j}\right)\left(\xi_{1}, \xi_{1}\right)=e^{2 \pi i\left(k_{2} \cdot \xi_{1}-k_{1} \cdot \xi_{2}\right)} Z \varphi_{j}(\xi)=0
$$

for all $k=\left(k_{1}, k_{2}\right) \in \mathbf{Z}^{2 d}$, and so the representation (10) implies that

$$
H_{k} f=\sum_{j=1}^{\infty} c_{j}\left\langle f, \pi(k) \varphi_{j}\right\rangle \pi(k) \varphi_{j}=0
$$

for all $k \in \mathbf{Z}^{2 d}$.

On the other hand $\left\langle H_{k} f, f\right\rangle=\int_{\mathrm{R}^{2 d}} T_{k} \sigma(z) \mathscr{V}_{\varphi} f(z)\langle\pi(z) \varphi, f\rangle d z=$ $\int_{\mathrm{R}^{2 d}} T_{k} \sigma(z)\left|\mathscr{V}_{\varphi} f(z)\right|^{2} d z=0$. Since $H_{k} f \in S_{0}, f \in S_{0}^{\prime}, \mathscr{V}_{\varphi} f \in L^{\infty}$, both sides are well-defined and coincide. Consequently, the continuous function $\mathscr{V}_{\varphi} f$ vanishes on the support of $T_{k} \sigma$. The main assumption (11) on $\sigma$ implies that $\bigcup_{k \in \mathrm{Z}^{2 d}} \operatorname{supp} T_{k} \sigma=\mathrm{R}^{2 d}$, and so $\mathscr{V}_{\varphi} f \equiv 0$ which is a contradiction to $f \not \equiv 0$. Hence, the $Z \varphi_{j}$ 's, $j \in \mathrm{N}$ cannot have common zeroes.

Finally we show with a compactness argument that there exists a finite subset $\{j=1, \ldots, r\} \subseteq \mathrm{N}$, such that $\bigcap_{j=1}^{r} \mathscr{Z}_{j}=\emptyset$. Now, in the compact set $[0,1]^{2 d}$ any family of closed sets with empty intersection contains a finite subfamily with empty intersection. Since we have already shown that $\bigcap_{j=1}^{\infty} \mathscr{Z}_{j}=\emptyset$, there exists a finite index $r$, such that $\bigcap_{j=1}^{r} \mathscr{Z}_{j}=\emptyset$. As a consequence, the $Z \varphi_{j}$, $j=1, \ldots, r$, do not have a common zero and thus $\sum_{j=1}^{r}\left|Z \varphi_{j}\right|^{2} \geq A>0$. This means that the set $\left\{\pi(k) \varphi_{j}: k \in Z^{2 d}, j=1, \ldots, r\right\}$ is a frame.

Proof of Theorem 3.I. We first show the norm-equivalence for $S_{0}$.

Assume first that $f \in S_{0}$. By the embedding $S_{0} \hookrightarrow L^{2}\left(\mathrm{R}^{d}\right)$ we have

$$
\left\|H_{k} f\right\|_{2} \leq C_{\varphi}\left\|H_{k} f\right\|_{S_{0}} .
$$

In the following chain of inequalities we use the fundamental estimate (8), Fubini-Tonelli for the interchange of summation and integration, and the hypothesis (11) on $\sigma$ in the final step. Hence,

$$
\begin{aligned}
\sum_{k}\left\|H_{k} f\right\|_{2} & \leq C_{\varphi} \sum_{k}\left\|H_{k} f\right\|_{S_{0}} \\
& =C_{\varphi} \sum_{k}\left\|\mathscr{V}_{\varphi}\left(\mathscr{V}_{\varphi}^{*}\left(T_{k} \sigma \mathscr{V}_{\varphi} f\right)\right)\right\|_{1} \\
& \leq C_{\varphi} C_{1} \sum_{k}\left\|T_{k} \sigma \mathscr{V}_{\varphi} f\right\|_{1} \\
& =C_{\varphi} C_{1} \int_{\mathrm{R}^{2 d}} \sum_{k} T_{k} \sigma(z)\left|\mathscr{V}_{\varphi} f(z)\right| d z \\
& \leq C_{\varphi} C_{1} B\|f\|_{S_{0}},
\end{aligned}
$$


so the mapping $f \rightarrow\left(\left\|H_{k} f\right\|_{2}\right)$ is bounded from $S_{0}$ to $\ell^{1}$.

Conversely, assume that $f \in S_{0}^{\prime}\left(\mathrm{R}^{d}\right)$ and that $\sum_{k}\left\|H_{k} f\right\|_{2}<\infty$. Since $\left\|H_{k} f\right\|_{2}=\sup _{\|g\|_{2}=1}\left|\left\langle H_{k} f, g\right\rangle\right|$, we have the inequality

$$
\sum_{k}\left|\left\langle H_{k} f, g_{k}\right\rangle\right| \leq \sum_{k}\left\|H_{k} f\right\|_{2}<\infty
$$

for arbitrary sequences $g_{k} \in L^{2}$ with $\left\|g_{k}\right\|_{2}=1$. Using the spectral representation of $H_{k}$ from Corollary 2.8, we then obtain

$$
\sum_{k}\left|\sum_{j=1}^{\infty} c_{j}\left\langle f, \pi(k) \varphi_{j}\right\rangle\left\langle\pi(k) \varphi_{j}, g_{k}\right\rangle\right| \leq \sum_{k}\left\|H_{k} f\right\|_{2} .
$$

We now fix $j_{0} \in \mathrm{N}$ and choose $g_{k}=\pi(k) \varphi_{j_{0}}$ for $k \in \mathrm{Z}^{2 d}$. Then

$$
\sum_{k}\left|\left\langle H_{k} f, g_{k}\right\rangle\right|=\sum_{k}\left|c_{j_{0}}\left\langle f, \pi(k) \varphi_{j_{0}}\right\rangle\right| \leq \sum_{k}\left\|H_{k} f\right\|_{2}<\infty .
$$

Since (17) holds for every $j_{0}$, we deduce that for every $r \in \mathrm{N}$

$$
\sum_{j=1}^{r} \sum_{k}\left|\left\langle f, \pi(k) \varphi_{j_{0}}\right\rangle\right| \leq\left(\sum_{j=1}^{r} \frac{1}{c_{j}}\right) \sum_{k}\left\|H_{k} f\right\|_{2} .
$$

According to Lemma 3.4 we can choose $r$ so large that the set $\left\{\pi(k) \varphi_{j}: k \in\right.$ $\left.\mathrm{Z}^{2 d}, j=1, \ldots, r\right\}$ is a frame for $L^{2}$. Furthermore, since $\varphi_{j} \in S_{0}$, Lemma 2.5 applies, and therefore (17) implies that $f \in S_{0}$ with a norm estimate

$$
\|f\|_{S_{0}} \leq C\left(\sum_{j=1}^{r} \frac{1}{c_{j}}\right) \sum_{j=1}^{r} \sum_{k}\left|\left\langle f, \pi(k) \varphi_{j}\right\rangle\right| .
$$

Combining the two estimates (16) and (18), we have shown that $\|f\|_{S_{0}}$ and $\sum_{k}\left\|H_{k} f\right\|_{2}$ are equivalent norms on $S_{0}$.

The statements about $L^{2}$ and $S_{0}^{\prime}$ are shown similarly and we only sketch the necessary modifications.

For the upper bound in the $L^{2}$-case, we use (7) again and proceed as in (16) to obtain

$$
\sum_{k}\left\|H_{k} f\right\|_{2}^{2} \leq \sum_{k}\left\|\mathscr{V}_{\varphi} \mathscr{V}_{\varphi}^{*}\left(T_{k} \sigma \mathscr{V}_{\varphi} f\right)\right\|_{2}^{2} \leq C_{\varphi} \sum_{k}\left\|T_{k} \sigma \mathscr{V}_{\varphi} f\right\|_{2}^{2}
$$

Now by hypothesis (11) on $\sigma$ we have

$$
\sum_{k} \int_{\mathrm{R}^{2 d}}\left|T_{k} \sigma(z)\right|^{2}\left|\mathscr{V}_{\varphi} f(z)\right|^{2} d z \leq B \int_{\mathrm{R}^{2 d}}\left|\mathscr{V}_{\varphi} f(z)\right|^{2} d z=B\|\varphi\|_{2}^{2}\|f\|_{2}^{2}
$$


Altogether we have obtained the upper bound

$$
\sum_{k}\left\|H_{k} f\right\|_{2}^{2} \leq C_{2}\|f\|_{2}^{2} .
$$

Assuming, conversely, that $\sum_{k \in Z^{2 d}}\left\|H_{k} f\right\|_{2}^{2}<\infty$ for $f \in S_{0}^{\prime}\left(\mathrm{R}^{d}\right)$, we may argue in analogy to the $S_{0}$-case and find that

$$
c_{j_{0}}^{2} \sum_{k}\left|\left\langle f, \pi(k) \varphi_{j_{0}}\right\rangle\right|^{2} \leq \sum_{k}\left\|H_{k} f\right\|_{2}^{2}<\infty .
$$

Now the assertion of Lemma 3.4 provides the lower bound, and thus we achieve the norm equivalence

$$
C_{1}\|f\|_{2}^{2} \leq \sum_{k \in \mathrm{Z}^{2 d}}\left\|H_{k} f\right\|_{2}^{2} \leq C_{2}\|f\|_{2}^{2} .
$$

Finally, assume that $f \in S_{0}^{\prime}$. Then again by (7)

$$
\sup _{k}\left\|\mathscr{V}_{\varphi}^{*}\left(T_{k} \sigma \mathscr{V}_{\varphi} f\right)\right\|_{2} \leq C \sup _{k}\left\|T_{k} \sigma \mathscr{V}_{\varphi} f\right\|_{2} \leq C\left\|\mathscr{V}_{\varphi} f\right\|_{\infty} \sup _{k}\left\|T_{k} \sigma\right\|_{2},
$$

so $\sup _{k}\left\|H_{k} f\right\|_{2} \leq C\|\sigma\|_{2}\|f\|_{S_{0}^{\prime}}$.

Conversely, if $\sup _{k \in Z^{2 d}}\left\|H_{k} f\right\|_{2}<\infty$, then again by diligent choice as in (17) we conclude that $c_{j_{0}} \sup _{k}\left|\left\langle f, \pi(k) \varphi_{j_{0}}\right\rangle\right| \leq \sup _{k}\left\|H_{k} f\right\|_{2}<\infty$ for all $j_{0}$. Using Lemma 2.5 we find that $\|f\|_{S_{0}^{\prime}} \leq C\left(\max _{j=1, \ldots, r} \frac{1}{c_{j}}\right) \sup _{k \in Z^{2 d}}\left\|H_{k} f\right\|_{2}$ for suitable $r \in \mathrm{N}$. This concludes the proof of all norm equivalences claimed in Theorem 3.1.

\section{An alternative model}

In principle one could study time-frequency localization operators with respect to other time-frequency representations. It is natural to guess that the form of the results will be similar to Theorem 3.1. In this section we show that the clean characterization of time-frequency concentration depends crucially on the type of time-frequency representation that occurs in the localization procedure. We will consider time-frequency localization via the Weyl calculus proposed as a model in [21]. The Weyl transform of a symbol $\sigma$ is often defined via the Wigner distribution, which is

$$
W f(x, \omega)=\int_{\mathrm{R}^{d}} f(x+t / 2) \overline{f(x-t / 2)} e^{-2 \pi i \omega \cdot t} d t
$$

for $f \in L^{2}$. The Wigner distribution is an optimal time-frequency representation with respect to several criteria and is still one of the most popular 
time-frequency representations. In practice, however, it is less attractive because of its lack of positivity and the disturbing presence of cross-terms in the representation of multi-component signals.

Since, similar to the isometry property of the STFT, the Wigner distribution satisfies $\int_{\mathrm{R}^{d}} \int_{\mathrm{R}^{d}} W f(x, \omega) d x d \omega=\|f\|_{2}^{2}$, one may define a family of localization operators for a symbol $\sigma$ on $\mathbf{R}^{2 d}$ and $k \in \mathrm{Z}^{2 d}$ by the quadratic form

$$
\left\langle L_{k} f, f\right\rangle=\left\langle T_{k} \sigma, W f\right\rangle .
$$

The resulting operators $L_{k}$ are exactly the Weyl transforms of $T_{k} \sigma$ and have been studied extensively in PDE and pseudodifferential operators, see [18] and [17], [22].

We show that in general the Wigner distribution is not suited for characterizing the time-frequency concentration of functions by means of time-frequency partitions. For this, we choose a symbol $\sigma$ in the form of a Wigner distribution, i.e. $\sigma=W \varphi$ for arbitrary $\varphi \in S_{0}$ with $\|\varphi\|_{2}=1$. Using Moyal's formula [20], we obtain that

$$
\left\langle L_{k} f, f\right\rangle=\langle W(\pi(k) \varphi), W f\rangle=\langle f, \pi(k) \varphi\rangle\langle\pi(k) \varphi, f\rangle,
$$

and thus $L_{k} f=\langle f, \pi(k) \varphi\rangle \pi(k) \varphi$ is just the orthogonal projection onto $\pi(k) \varphi$.

We now repeat the construction in the proof of Lemma 3.4. Since $\varphi \in S_{0}$, its Zak transform has a zero $\xi \in[0,1]^{2 d}$ by Proposition 2.3iv). The associated linear functional $f \in S_{0}^{\prime}$ defined as $\langle f, \varphi\rangle=Z \varphi(\xi)$ satisfies

$$
\langle f, \pi(k) \varphi\rangle=0 \quad \forall k \in Z^{2 d} .
$$

Thus we have constructed a non-zero distribution $f \in S_{0}^{\prime}\left(\mathrm{R}^{d}\right)$ such that

$$
\sum_{k}\left\|L_{k} f\right\|_{2}=\sum_{k}|\langle f, \pi(k) \varphi\rangle|=0 .
$$

Hence, unlike the assertion of Theorem 3.1, the condition $\sum_{k}\left\|L_{k} f\right\|_{2}<\infty$ cannot characterize $S_{0}$.

REMARK 4.1. The above counter-example is related to the amalgam version of the Balian-Low theorem, [1], [11], which states that $\mathscr{G}\left(\varphi, \mathrm{Z}^{2 d}\right)$ cannot be a frame for $L^{2}\left(\mathrm{R}^{d}\right)$ when $\varphi \in S_{0}$. In the language of localization operators, this means that the expression

$$
\sum_{k}\left\|L_{k} f\right\|_{2}^{2}=\sum_{k}|\langle f, \pi(k) \varphi\rangle|^{2}
$$

is not equivalent to the $L^{2}$-norm. 
REMARK 4.2. Theorem 3.1 can be generalized to lattices $\Lambda$ other than $Z^{d} \times$ $\mathrm{Z}^{d}$. If $\Lambda=a \mathbf{Z}^{d} \times b \mathbf{Z}^{d}$ and $(a b)^{-1} \in \mathbf{N}$ (integer oversampling) or $a b \in \mathbf{Q}$ (rational oversampling), then the Zak transform methods of Sections 2 and 3 could be modified to prove a version of Theorem 3.1. However, the case of general lattices, where either $a b \notin \mathrm{Q}$ or $\Lambda$ is not separable, is beyond Zak transform methods.

Furthermore, one may want to characterize the whole class of modulation spaces (they measure the time-frequency concentration with more refined norms than $S_{0}$ and $S_{0}^{\prime}$ ) in the style of the main theorem. Both extensions require completely different methods and will be presented in a separate work [9].

\section{REFERENCES}

1. Benedetto, J. J., Heil, C., and Walnut, D. F., Gabor systems and the Balian-Low theorem, in Gabor analysis and algorithms, pp. 85-122, Birkhäuser Boston, Boston, MA, 1998.

2. Berezin, F. A., Wick and anti-Wick symbols of operators, Mat. Sb. (N.S.) 86(128) (1971), 578-610.

3. Boggiatto, Paolo, and Cordero, Elena, Anti-Wick quantization with symbols in $L^{p}$ spaces, Proc. Amer. Math. Soc. 130(9) (2002), 2679-2685 (electronic).

4. Cordero, Elena, and Gröchenig, Karlheinz, Time-frequency analysis of localization operators, J. Funct. Anal. 205(1) (2003), 107-131.

5. Córdoba, A., and Fefferman, C., Wave packets and Fourier integral operators, Comm. Partial Differential Equations 3(11) (1978), 979-1005.

6. Daubechies, I., The wavelet transform, time-frequency localization and signal analysis, IEEE Trans. Inform. Theory 36(5) (1990), 961-1005.

7. Daubechies, I., Ten lectures on wavelets, Society for Industrial and Applied Mathematics (SIAM), Philadelphia, PA, 1992.

8. De Mari, F., Feichtinger, H. G., and Nowak, K., Uniform eigenvalue estimates for timefrequency localization operators, J. London Math. Soc. (2) 65(3) (2002), 720-732.

9. Dörfler, M., and Gröchenig, K., Time-frequency partitions on general lattices for modulation spaces, in preparation.

10. Feichtinger, H. G., On a new Segal algebra, Monatsh. Math. 92(4) (1981), 269-289.

11. Feichtinger, H. G., and Gröchenig, K., Gabor frames and time-frequency analysis of distributions, J. Funct. Anal. 146(2) (1997), 464-495.

12. Feichtinger, H. G., and Kozek, W., Quantization of TF lattice-invariant operators on elementary LCA groups, in Gabor analysis and algorithms, pp. 233-266, Birkhäuser Boston, Boston, MA, 1998.

13. Feichtinger, H. G., and Strohmer, T., editors, Gabor Analysis and Algorithms: Theory and Applications, Birkhäuser Boston, Boston, MA, 1998.

14. Feichtinger, Hans G., and Nowak, Krzysztof, A first survey of Gabor multipliers, in Advances in Gabor analysis, Appl. Numer. Harmon. Anal., pp. 99-128, Birkhäuser Boston, Boston, MA, 2003.

15. Feichtinger, H. G., and Zimmermann, G., A Banach space of test functions for Gabor analysis, in H. G. Feichtinger and T. Strohmer, editors, Gabor Analysis and Algorithms: Theory and Applications, pp. 123-170, Chap. 3, Birkhäuser, Boston, 1998.

16. Gel' fand, I. M., and Vilenkin, N. Ya., Generalized Functions. Vol. 4, Academic Press [Harcourt Brace Jovanovich Publishers], New York, 1964 [1977]. Applications of harmonic analysis. Translated from the Russian by Amiel Feinstein. 


\section{MONIKA DÖRFLER, HANS G. FEICHTINGER AND KARLHEINZ GRÖCHENIG}

17. Gröchenig, Karlheinz, Foundations of Time-frequency Analysis, Birkhäuser Boston Inc., Boston, MA, 2001.

18. Hörmander, Lars, The Analysis of Linear Partial Differential Operators III, Pseudodifferential operators, Grundlehren Math. Wiss. [Fundamental Principles of Mathematical Sciences] 274.

19. Janssen, A. J. E. M., The Zak transform: a signal transform for sampled time-continuous signals, Philips J. Res. 43(1) (1988), 23-69.

20. Moyal, J. E., Quantum mechanics as a statistical theory, Proc. Cambridge Philos. Soc. 45 (1949), 99-124.

21. Ramanathan, J., and Topiwala, P., Time-frequency localization via the Weyl correspondence, SIAM J. Math. Anal. 24(5) (1993), 1378-1393.

22. Wong, M. W., Localization operators on the Weyl-Heisenberg group, in Geometry, analysis and applications (Varanasi, 2000), pp. 303-314, World Sci. Publishing, River Edge, NJ, 2001.

INSTITUT FÜR MATHEMATIK

UNIVERSITÄT WIEN

STRUDLHOFG. 4

A-1090 WIEN

AUSTRIA

E-mail: monika.doerfler@univie.ac.at

E-mail: hans.feichtinger@univie.ac.at
INSTITUTE OF BIOMATHEMATICS AND BIOMETRY GSF - NATIONAL RESEARCH CENTER FOR ENVIRONMENT AND HEALTH INGOLSTÄDTER LANDSTRAßE 1 85764 NEUHERBERG GERMANY

E-mail: karlheinz.groechenig@gsf.de 